\title{
Numerical Analysis of Two-phase Flow and Heat Transfer in External Cyclic Purification Hot-dip Galvanizing Bath
}

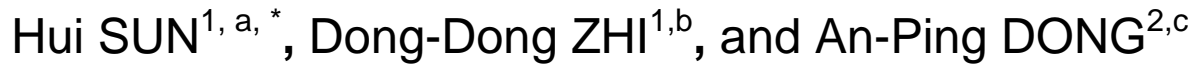 \\ ${ }^{1}$ College of Mechanical Engineering, Shanghai Dianji University, Shanghai 201306, China \\ ${ }^{2}$ State Laboratory of Metal Matrix Composites, Shanghai Jiaotong University, Shanghai 200240, \\ China \\ ${ }^{a}$ sunh@sdju.edu.cn, b'zhidy.1813@163.com, capdong@sjtu.edu.cn \\ ${ }^{*}$ Corresponding author
}

Keywords: Hot-dip galvanizing bath, External cyclic purification, Numerical simulation, Dross concentration

\begin{abstract}
The mixture model based on the Euler-Euler approach along with the standard $k-\varepsilon$ turbulent model was employed to predict the two-phase flow and heat transfer in the external cyclic purification hot-dip galvanizing bath. The arrangement of the return and pump pipes was assessed to determine its effect on the flow and dross concentration distribution in the bath. The results show that, the level of the dross concentration in the external cyclic purification galvanizing bath gradually decreases with processing time, and the dross concentration on the surfaces of the steel strip is evenly distributed. The arrangement of the return and pump pipes has an important influence on the flow in $\mathrm{V}$ region and its vicinity, and the dross concentration distribution on the strip surfaces. The scheme that the return and pump pipes are both placed on the outside of the steel strip exit section is the optimal.
\end{abstract}

\section{Introduction}

Hot-dip galvanized steel has been an engineering material for many industries such as automobile, home appliance and construction industries[1-4]. Improvement of its quality has currently become a major focus of researchers. The operating conditions of the steel strip and molten zinc in continuous hot-dip galvanizing bath are the most important factors that affect the coating quality of galvanized steel sheet. Because of a number of problems such as high-temperature operation, on-line measurement difficulties, and high cost occurred in the experimental study, computational fluid dynamics (CFD) method has been widely employed. Recently, Ajersch and Ilinca[5-6] simulated the flow and heat transfer in the galvanizing bath, and evaluated the effects of line speed, strip width, strip temperature and ingot addition on the velocity and temperature fields in the bath. They also simulated the spatial distribution of compositional variation in the hot-dip galvanizing bath[7-8]. Dash et al.[9] simulated the flow structure in a continuous hot-dip galvanizing bath. They set the flow barriers in the V region to eliminate the vortex formed between the strip and the sink roll[9]. Chen et al.[1] studied numerically the flow and heat transfer in the Galvalume bath under different speeds, widths and entry temperatures of the steel strip. However, the current research has mainly been focused on the flow and heat transfer of molten zinc. Very often, the dross concentration distribution in the galvanizing bath has little been presented. In addition, the research is mostly limited to the traditional hot-dip galvanizing bath. The goal of this research is to predict numerically the two-phase flow and heat transfer in a novel external cyclic purification hot-dip galvanizing bath, and investigate the influence of different arrangements of the return and pump pipes on the flow and dross concentration fields, which can be used as the basis for the industrial application of this novel hot-dip galvanizing bath. 


\section{System Geometry}

The model system studied in this work is a novel external cyclic purification hot-dip galvanizing bath, which is mainly developed by Shanghai Jiaotong University (see Fig.1). The electromagnetic purification and external circulation devices are equipped on the traditional hot-dip galvanizing bath. The electromagnetic purification device is composed of a ceramic separator and an electrified coil. The coil is switched on, and the induced alternating magnetic field is created in the ceramic separator. The zinc dross bears the repulsive force opposite to the electromagnetic force on the melt, and the repulsive force is much less than the electromagnetic forces for the same volume of zinc melt. In consequence, the dross particles can be successfully separated from the metal melt. The external circulation device consists of a zinc pump, a return pipe and a pump pipe, which can implement the external circulation of the molten zinc in the bath. The electromagnetic purification device is connected with the zinc pump, and the return and pump pipes are separately linked with the electromagnetic purification device and the zinc pump. In operation, the zinc pump delivers the molten zinc through the pump pipe from the galvanizing bath to the ceramic separator for electromagnetic purification, and then the purified molten zinc flows back to the bath through the return pipe.
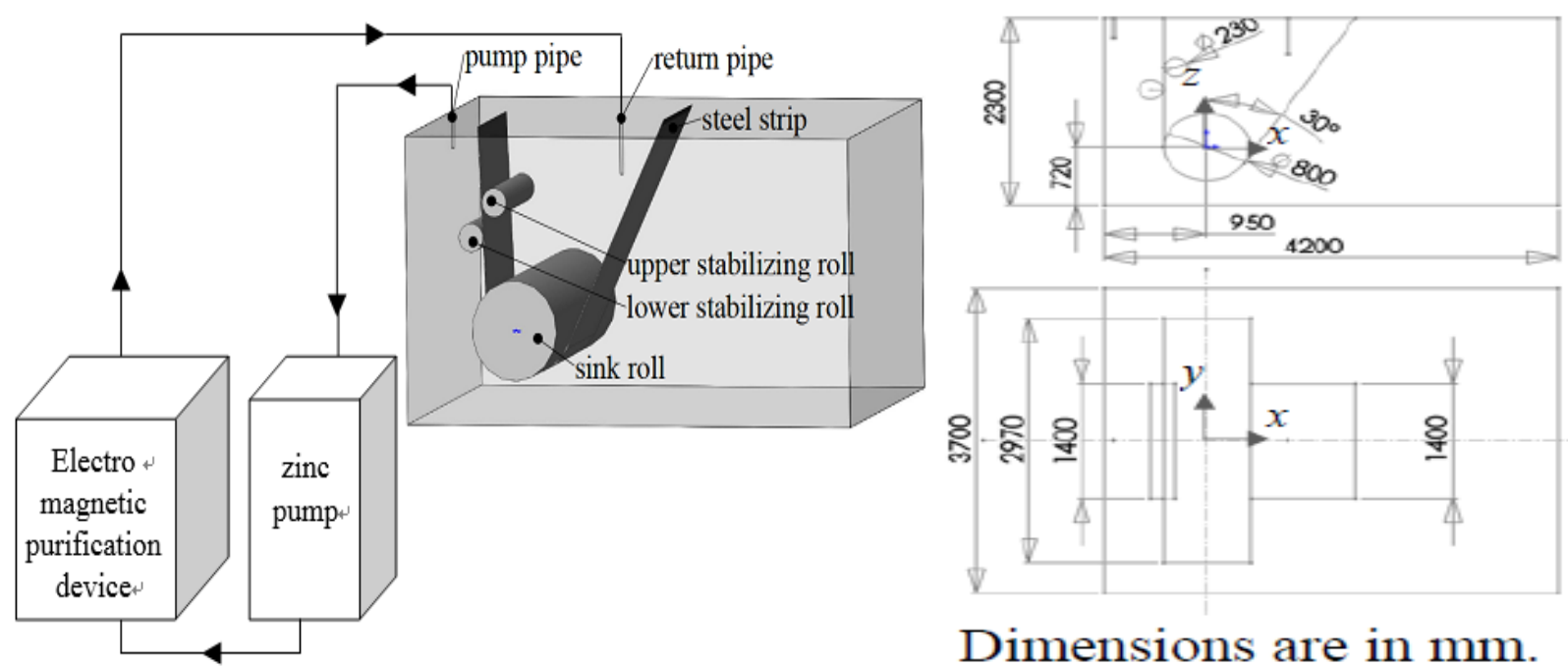

\section{Dimensions are in $\mathrm{mm}$.}

Fig.1 Schematic diagram of the external cyclic purification hot-dip galvanizing bath

Fig. 1 also shows the geometry of the galvanizing bath, which confirms to a real life industrial size galvanizing process. The return and pump pipes are arranged at the top surface of the galvanizing bath, and their inner diameters are both $30 \mathrm{~mm}$. In order to analyze the influence of the arrangement of return and pump pipes on the flow and heat transfer in the galvanizing bath, several different schemes (P1 P3 model, see Fig.2) are designed.

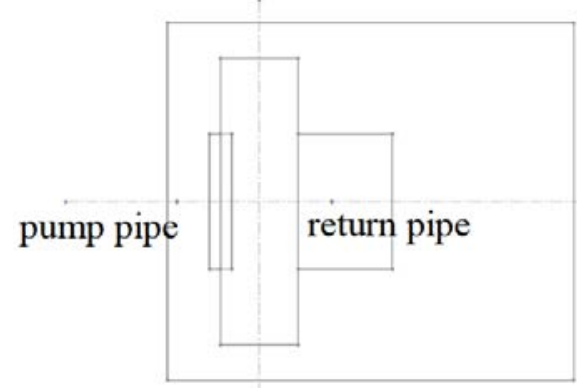

P1 model

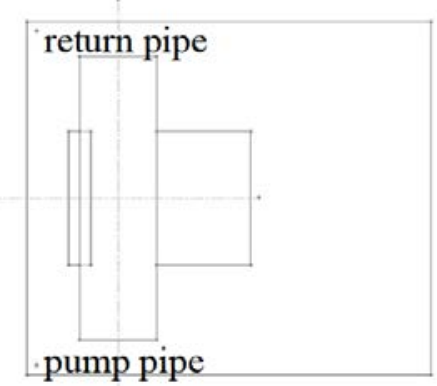

P2 model

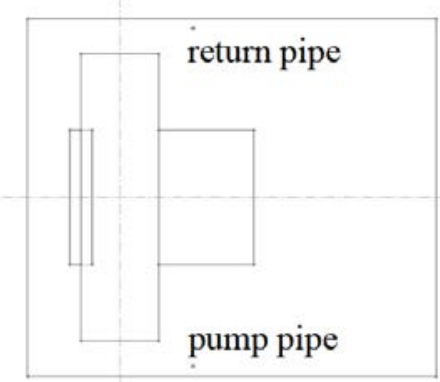

P3 model

Fig.2 Arrangement of the return and pump pipes in the hot-dip galvanizing bath 


\section{CFD Analysis Method}

\section{Mathematical Model}

The mixture model based on the Euler-Euler approach along with the standard $k-\varepsilon$ turbulent model is employed to predict the two-phase flow and heat transfer in the external cyclic purification hot-dip galvanizing bath. Balancing the accuracy and computational cost, the bath model is set up based on the following assumptions: (1) The molten zinc is incompressible. (2) The dross formation is ignored, and the diameter of the dross is taken as a constant. (3) The steel strip is assumed as a zero-thickness wall to control the grid size effectively. (4) It is well known that the steel strip is tangent to the sink roll and there are small angles between the steel strip and the stabilizing rolls. In order to avoid excessively distorted elements in these regions during grid generation, the steel strip is divided into several segments and moved up or down a short distance from the contact positions.

\section{Grid Generation and Model Settings}

The Delaunay triangulation method is employed to mesh the flow domain. The molten zinc is specified as primary phase and the dross as secondary phase. The physical parameters for all phases are given in Table 1 , and the diameter of the dross is taken as a constant equal to $20 \mu \mathrm{m}$. The slip velocity is specified as Manninen-et-al model[10].

Table 1 Physical parameters of the molten zinc and the dross

\begin{tabular}{lllll}
\hline & $\begin{array}{l}\text { Density } \\
{\left[\mathrm{kg} / \mathrm{m}^{3}\right]}\end{array}$ & $\begin{array}{l}\text { Viscosity } \\
{[\mathrm{Pa} \cdot \mathrm{s}]}\end{array}$ & $\begin{array}{l}\text { Specific heat } \\
{[\mathrm{J} /(\mathrm{kg} \cdot \mathrm{K})]}\end{array}$ & $\begin{array}{l}\text { Thermal conductivity } \\
{[\mathrm{W} /(\mathrm{m} \cdot \mathrm{K})]}\end{array}$ \\
\hline molten zinc & 6600 & 0.00385 & 512 & 60 \\
dross & 7250 & $\ldots$ & 512 & 60 \\
\hline
\end{tabular}

The boundary conditions are as follows: The no-slip wall condition with standard wall-function is assumed at the bath walls, steel strip, sink roll and stabilizing rolls, and the temperature is set as 738K. Considering that the steel strip is moving in the direction of its path, the moving speed is specified for all the mobile surfaces. Considering the top surface of the galvanizing bath is covered by the dross, the no-slip stationary wall condition is imposed on the top surface. The thermal boundary of the top surface is treated as a combined boundary, that is, both the external radiation and the convective are simultaneously considered. At the inlet of return pipe, the inlet-velocity boundary is adopted. The outflow boundary is assumed at the outlet of the pump pipe. The initial volume fraction of the dross in the external cyclic purification galvanizing bath is set as $0.12 \%$. The governing equations are solved by adopting the finite-volume method. The SIMPLE algorithm is employed to couple the continuity and momentum equations. The first order upwind discretization scheme is used for the convective terms. The converge solution is assumed when the scaled residuals of all variables are smaller than $10^{-5}$, and the rate of the molten zinc entering and leaving must be equal.

\section{Results and Discussions}

\section{Flow Fields Analysis}

The comparison of the flow fields produced in the traditional and external cyclic purification galvanizing baths is shown in Fig.3. The selected vertical cross section is the $x-z$ plane at $y=0 \mathrm{~mm}$. For the external cyclic purification galvanizing bath, two cases are included, that is, the return pipe is placed in the V region (P1 model) or on the outside of the steel strip exit section (P2 model). 


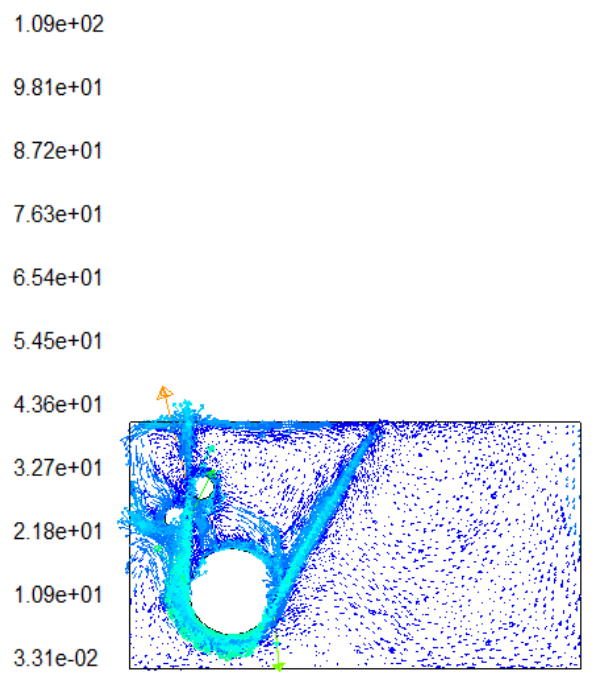

traditional bath

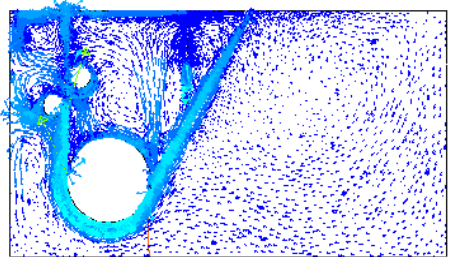

P1 model

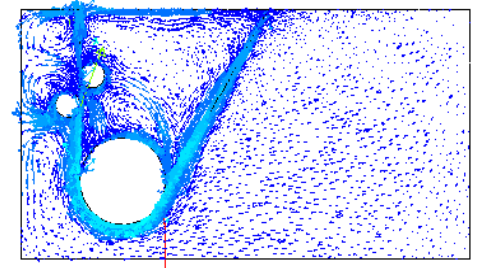

P2 model

Fig.3 Comparison of the flow fields produced in the traditional and external cyclic purification galvanizing baths

It can be seen that, the driving force of the molten zinc flow in the bath is the movement of the steel strip and the rotation of the sink roll and two stabilizing rolls. The return and pump pipes mainly affect the flow in the $\mathrm{V}$ region and its vicinity, and have little effect on the bulk flow in the bath. In addition, there is little variation in the flow near the top surface, which indicates that the return and pump pipes would not intensify the fluctuation on the top surface of the molten zinc, and thus would not spoil the coating quality of galvanized steel sheet.

When the return pipe is placed in the $\mathrm{V}$ region ( $\mathrm{P} 1$ model), it mainly affects the top vortex in the $\mathrm{V}$ region. The vortex center position and size change significantly, and the circulation of the molten zinc in the $\mathrm{V}$ region is accelerated. The ejected molten zinc flows toward the sink roll, then the flow is deflected after confronting with the sink roll and stabilizing rolls, and finally the molten zinc flows to the exit side of the steel strip. Because the molten zinc is purified by the electromagnetic purification device, the dross concentration in the vicinity of the steel strip in the external cyclic purification galvanizing bath is lower than that of the traditional bath, which is beneficial to the quality of the hot-dip galvanizing steel sheet.

When the return pipe is placed on the outside of the steel strip exit section (P2 model), it mainly affects the top vortex at the outside of the strip exit section.

This vortex is near the top surface of the bath, which can bring the dross particles from the top surface back to the flow again so that the steel strip could pick up the dross thus spoiling its own quality. For the external cyclic purification galvanizing bath, when placing the return pipe on the outside of the steel strip exit section, the vortex moves upward slightly, but its strength decreases. In consequence, the dross pick by the vortex can be less and the level of the dross concentration in the exit section of the steel strip drops, which is also beneficial to the quality of the hot-dip galvanizing steel sheet.

\section{Dross Concentration Fields Analysis}

\section{Global Dross Concentration and Dross Removal Efficiency}

The dross concentration field in the external cyclic purification galvanizing bath at different times is shown in Fig.4. Because of the similarity, only the results of P1 model are given here. For comparison, only the dross concentration within the range from 0.0007 to 0.0012 is displayed. 


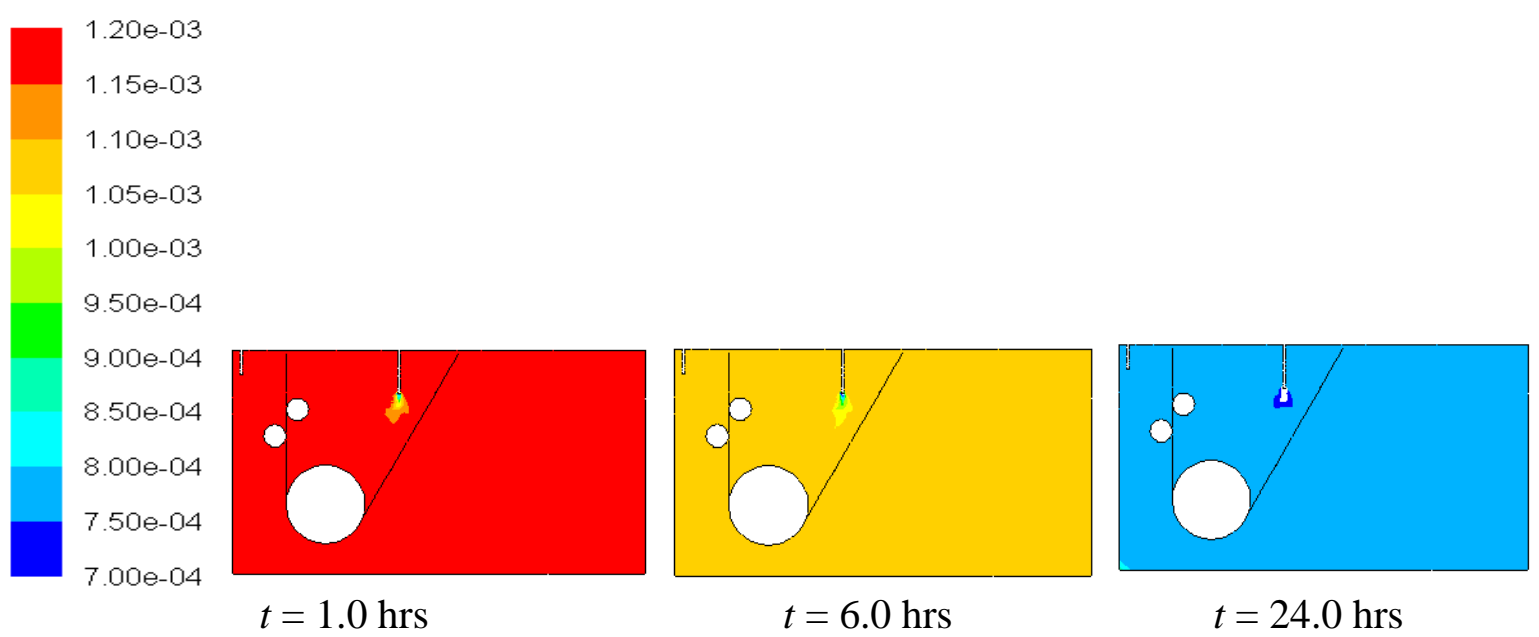

Fig.4 Dross concentration field in the external cyclic purification galvanizing bath at different processing times (P1 model)

It is clear from the above figure that, the dross concentration in the bath decreases with processing time. Except for the regions near the return pipe, the dross concentration in the bath is relatively evenly distributed. To further investigate the dross concentration in the external cyclic purification galvanizing bath at different times, the dross removal efficiency $\alpha$ is defined and calculated:

$$
\alpha=\frac{Q}{Q_{0}} \times 100 \%
$$

where, $Q$ is the dross removal amount, and $Q_{0}$ is the initial dross content.

The dross removal efficiency under the condition of external cyclic purification at different processing times is given in Table 2. The dross removal efficiency gradually increases with the processing time. There is very little difference in the dross removal efficiency for different arrangements of the return and pump pipes. All these coincide with the analysis results of the global dross concentration distribution in the galvanizing bath.

Table 2 Dross removal efficiency under the condition of external cyclic purification (\%)

\begin{tabular}{lllllll}
\hline Time[hrs] & 1.0 & 5.0 & 9.0 & 12.0 & 18.0 & 24.0 \\
\hline P1 model & 1.79 & 8.41 & 14.85 & 19.39 & 27.73 & 35.21 \\
P2 model & 1.81 & 8.48 & 14.97 & 19.52 & 27.90 & 35.42 \\
P3 model & 1.81 & 8.52 & 15.01 & 19.57 & 27.96 & 35.49 \\
\hline
\end{tabular}

\section{Local Dross Concentration Analysis}

Fig. 5 is an expanded view of the local dross concentration in V region under the condition of external cyclic purification. The processing time $t=24$ hrs. Fig.6 illustrates the dross concentration distributions on the surfaces of the steel strip in the external cyclic purification galvanizing bath with different schemes. It should be noted that, there are two narrow gaps on the surfaces of the steel strip exit section shown as in Fig.6(b), which are the results of geometry modelling. As mentioned above, in order to avoid excessively distorted elements, the steel strip exit section is divided into three segments and moved up or down a short distance from the contact positions during modelling. 

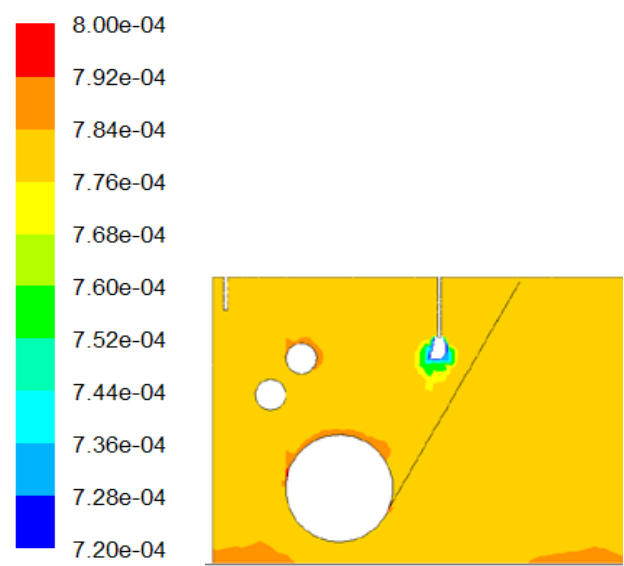

P1 model

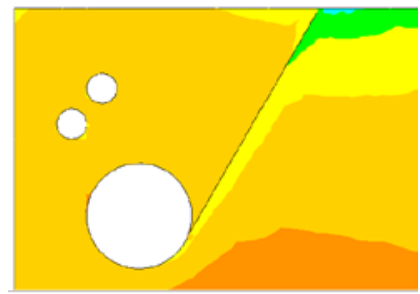

P2 model

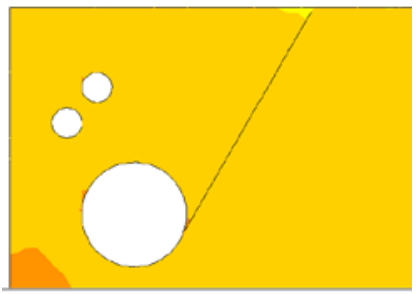

P3 model

Fig.5 An expanded view of the local dross concentration in V region under the condition of external cyclic purification

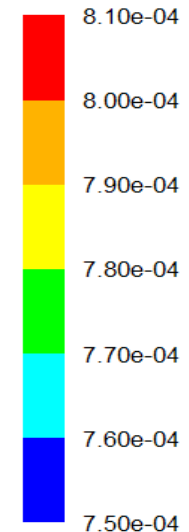

$7.50 \mathrm{e}-04$

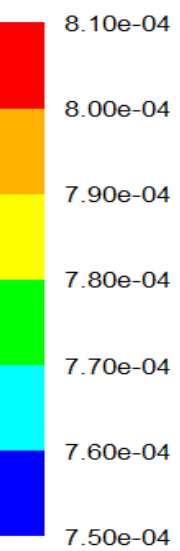

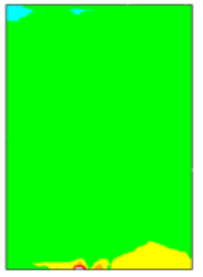

P1 model

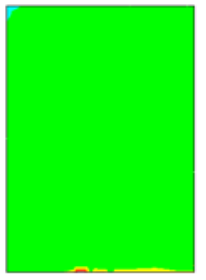

(a) surfaces of the steel strip entry section

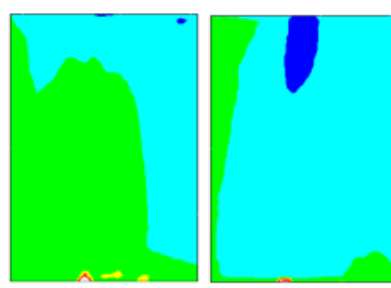

P2 model

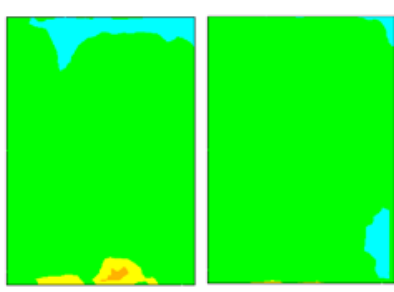

P3 model 
sedimentation occurs on the sink roll when P1 model is adopted. Therefore, P2 model is the best scheme, followed by P3 model, and P1 model is not a good choice.

\section{Conclusions}

The level of the dross concentration in the external cyclic purification hot-dip galvanizing bath gradually decreases with processing time, and the dross concentration on the surfaces of the steel strip is evenly distributed, which is advantageous to ensure the uniformity of the coating and improve the quality of the hot-dip galvanized steel sheet.

The return and pump pipes mainly affect the flow in the $\mathrm{V}$ region and its vicinity, and have little effect on the bulk flow in the galvanizing bath. They also would not intensify the fluctuation on the top surface of the molten zinc, and thus would not spoil the coating quality of galvanized steel sheet.

The arrangement of the return and pump pipes has an important influence on the dross concentration distribution on the strip surfaces. The scheme that the return and pump pipes are both placed on the outside of the steel strip exit section is the optimal.

\section{Acknowledgements}

The authors gratefully acknowledge the financial supports from National Natural Science Foundation of China(51304133, 51204110).

\section{References}

[1] Hairui CHEN, Haoping PENG, Xuping SU, et al: Transactions of Materials and Heat Treatment Vol 36(2015) , p. 223 (in Chinese).

[2] Jianhua DU, Yongli JIN, Yongfu WU, et al: Research on Iron \& Steel Vol 40(2012) , p. 26 (in Chinese).

[3] Xichuan CAI, Anping DONG, Da SHU, et al: Materials for Mechanical Engineering Vol 32(2008) , p. 1 (in Chinese).

[4] Yong Hee KIM, Young Whan CHO, Soon-Hyo CHUNG, et al: ISIJ International Vol 40(2000), p. 706.

[5] F. AJERSCH, F. ILINCA, J. F. HETU: Metallurgical and Materials Transactions B Vol 35(2004), p. 161.

[6] F. ILINCA, J. F. HETU: Numerical Heat Transfer Part A Vol 44(2003) , p. 463.

[7] F. AJERSCH, F. ILINCA, J. F. HETU, et al: Canadian Metallurgical Quarterly Vol 44(2005), p. 369.

[8] F. ILINCA, J. F. HETU, F. AJERSCH: Iron and Steel Technology Vol 1(2004) , p. 1067.

[9] Sukanta K. DASH, Monojit DUTTA, N. RAJESH: ISIJ International Vol 45(2005) , p. 1059.

[10] M. Manninen, V. Taivassalo, S. Kallio. On the mixture model for multiphase flow. VTT Publications 288, Technical Research Centre of Finland, 1996. 